\title{
The Relationship between the Extent of Collaboration of General Practitioners and Pharmacists and the Implementation of Recommendations Arising from Medication Review
}

\author{
A Systematic Review
}

\author{
Henk-Frans Kwint • Lynette Bermingham • \\ Adrianne Faber • Jacobijn Gussekloo • \\ Marcel L. Bouvy
}

(C) Springer International Publishing Switzerland 2013

\begin{abstract}
Background Many studies have investigated the effect of medication review on a variety of outcomes, but the elements of the interventions have been quite diverse. Moreover, implementation rates of recommendations also vary widely between studies.

Objective The objective of this study was to investigate how the extent of collaboration between the general practitioner (GP) and the pharmacist impacts on the implementation of recommendations arising from medication review.

Methods MEDLINE, EMBASE and Web of Science were searched for studies published between January 2000 and April 2012. Keywords included medication review, medication therapy management, pharmaceutical services and drug utilization review. Sixteen articles (describing 14 randomized controlled trials [RCTs]) out of 620 titles met the inclusion criteria. Inclusion criteria for the review were medication review, RCT design, involvement of both
\end{abstract}

Electronic supplementary material The online version of this article (doi:10.1007/s40266-012-0048-6) contains supplementary material, which is available to authorized users.

H.-F. Kwint $(\bowtie) \cdot$ M. L. Bouvy

Division of Pharmacoepidemiology \& Clinical Pharmacology,

Utrecht Institute for Pharmaceutical Sciences (UIPS),

PO Box 80082, 3508 TB Utrecht, The Netherlands

e-mail: h.f.kwint@uu.nl

H.-F. Kwint · A. Faber · M. L. Bouvy

SIR Institute for Pharmacy Practice and Policy,

Leiden, The Netherlands

L. Bermingham · J. Gussekloo

Department of Public Health and Primary Care, Leiden

University Medical Center, Leiden, The Netherlands pharmacist and GP, and home-dwelling patients (mean age $>70$ years) who had not been recently discharged. After quality assessment of the article, the presence of the following eight key elements reflecting collaboration were scored for each intervention: pharmacist with clinical experience, own pharmacist involved, sharing of medical records, patient interview by pharmacist, invitation of patients by GP, case conference between GP and pharmacist, action plan, follow-up. The primary outcome was the implementation rate of recommendations. Meta-regression analysis was used to assess the association between the implementation rate and the number of key elements present.

Results Twelve RCTs were included after quality assessment. The mean number of key elements within the intervention was 5.2 (range 1-8). The mean implementation rate of recommendations was $50 \%$ (range 17-86). The association between the number of key elements present in the intervention and the implementation rate of recommendations was significant: $\beta=0.085 \quad(95 \% \quad \mathrm{CI}$ 0.052-0.128; $p<0.0001)$.

Conclusion This systematic review shows a significant association between the number of key elements of the intervention reflecting collaborative aspects in medication review and the implementation rate of recommendations.

\section{Introduction}

Polypharmacy and drug-related morbidity is increasingly recognized as a major public health problem among the elderly $[1,2]$. Medication review has been proposed as an important strategy to constrain the negative effects of polypharmacy, aiming at safer and more effective use of medicines $[3,4]$. 
Medication review has been defined as "a structured, critical examination of a patient's medicines with the objective of reaching an agreement with the patient about treatment, optimising the impact of medicines, minimizing the number of medication-related problems and reducing waste" [5]. Three types of medication review have been described, based on the purpose of the review: 'prescription review' [technical issues related to prescription(s)], 'concordance or compliance review' (issues relating to the patient's medicine behaviour) and 'clinical medication review' (issues relating to the patient's use of medicines in the context of their condition) [6]. Concomitantly, efforts have been made to standardize medication review [5-7].

However, systematic reviews of pharmacist-led medication review have not shown an effect on clinical outcomes such as hospital admissions or mortality [8-10]. In some studies, positive effects were reported on intermediate outcomes like drug knowledge and adherence [8]. The heterogeneity in patient populations, settings, interventions and outcomes in these studies made it difficult to draw definitive conclusions. There may be merit in combining the expertise of the pharmacist and physician with shared decision-making involving the patient in order to improve outcomes [11]. Previous systematic reviews did not take into account the variability in collaboration between pharmacists and general practitioners (GPs) in medication reviews.

Studies on barriers and facilitators in medication review reveal collaborative aspects that might be essential for conducting successful medication reviews [12-14]. The most commonly cited facilitators were having an established pharmacist-physician relationship [13, 14] and a face-to-face meeting (case conference) between pharmacist and physician to discuss the pharmacist's recommendations $[13,15,16]$. Using a pharmacist other than the patient's regular pharmacist was seen as a barrier, as was inadequate clinical training of the pharmacist [13]. Without access to medical records, the pharmacist may make tentative or inappropriate recommendations that are of little help [13]. The GPPC (General Practitioner-Pharmacist Collaboration) study [17] further suggested that a general practicebased service could be more facilitating than a community pharmacy-based service [17]. This could imply that patients are approached for medication review by the GP practice, which is also common in the Home Medicines Review (HMR) programme in Australia [12, 13]. They further suggested that the pharmacist should meet the patient for interview about their medicines in the physician's office [17-19], while a patient interview at home by an accredited pharmacist is the predominant step of the HMR programme $[12,13]$. Finally, it is important for a collaborative medication review that responsibilities for implementation of the action plan and follow-up are clearly defined and divided between physician and pharmacist [13].

The aim of this systematic review was to investigate how the extent of collaboration between the GP and the pharmacist impacts on the implementation of recommendations arising from medication review.

\section{Methods}

\subsection{Search}

Our search strategy identified research on medication review interventions involving pharmacists and GPs. MEDLINE, EMBASE and Web of Science were searched for articles published between 1 January 2000 and 1 April 2012. These dates were chosen because relatively few studies with an elaborate description of the medication review process were published before 2000. Interventions were identified using the following keywords and medical subject headings $(\mathrm{MeSH})$ : medication review, medication therapy management, pharmaceutical services and drug utilization review (see Appendix S1 for detailed search terms [Online Resource 1]). Different publications on the same group of patients were considered as one study.

\subsection{Study Selection}

All titles were reviewed by two investigators (H.K. and L.B.). Studies were excluded if both agreed that the title clearly indicated that the study did not concern medication review and/or focussed on only one drug or drug class. H.K. and L.B. assessed all remaining abstracts independently in this manner. Studies were included if they fulfilled the following criteria: medication review, randomized clinical trial (RCT), pharmacist and GP involved, home-dwelling patients in primary care, mean age $\geq 70$ years, patients not recently discharged ( $<1$ month).

Only studies in English were included. Finally, full papers from potential studies were assessed independently by the two investigators for their suitability for inclusion. Differences were resolved by discussion, or a third investigator (either A.F. or M.B.) was consulted.

\subsection{Quality Assessment of the Studies}

Trial quality was assessed according to the Delphi list [20]. This list consists of ten criteria: randomization, treatment allocation, similar groups at baseline, eligibility criteria, blinding of outcome assessor, blinding of care provider, blinding of patient, point estimates and measures of variability, intention-to-treat analysis and reporting of 
withdrawal/drop-out rate. In addition, we added 'power calculation' to this list. Studies with a low score on the quality assessment ( 5 or fewer items scored 'yes') were excluded for analysis of outcomes.

\subsection{Study Characteristics}

\subsubsection{Categorization}

Studies were categorized by study author, year of publication, number of pharmacists and GPs, country, number of patients, duration of the study, mean age and sex of patients, mean number of drugs, description of the intervention, setting, number of recommendations in the intervention group, the clinical, intermediate and process outcomes assessed and the quality score.

\subsubsection{Outcomes}

The primary outcome was the implementation rate of recommendations following drug-related problems (DRPs) identified during medication review. The implementation rate was defined as the percentage of recommendations fully or partly implemented and/or the percentage of DRPs resolved. Partial implementation of recommendations means that an action other than that originally proposed by the pharmacist was implemented. Fully and partly implemented recommendations were counted equally. Data on clinical outcomes (hospital admissions, quality of life), intermediate outcomes (adherence) and other process outcomes (drug changes, number of drugs) were also extracted. The effect on clinical, intermediate and process outcomes was described as a significant effect in favour of the intervention group, a significant effect in favour of the control group or no significant effect.

\subsubsection{Key Elements of the Intervention}

The intervention was characterized by the presence or absence of eight key elements reflecting collaborative aspects between a GP and a pharmacist, based on the aforementioned facilitators and barriers in medication review [12-14]. The choice of the key elements was supported by scientific discussion with experienced pharmacist reviewers who regarded these elements as having 'face validity'. The following key elements were assessed: (1) 'pharmacist with clinical experience' means that the study pharmacist had adequate clinical training and expertise to perform medication reviews; (2) 'own pharmacist involved' means that the study pharmacist is the patient's regular pharmacist who has a longer lasting therapeutic relationship with his or her patient; (3) 'sharing of medical records' describes full access for the care provider performing the medication review to GP data on diseases of the patient and clinical values; (4) 'patient interview by pharmacist' means a face-to-face consultation between a pharmacist and a patient-this pharmacist must have a relationship with the GP; (5) 'invitation of the patients by GP' means that the patient is invited to the study or referred for medication review by the GP (practice); (6) "case conference GP and pharmacist' indicates a face-to-face meeting between at least the GP and the pharmacist to discuss the DRPs and recommendations for specific patients; (7) 'action plan' means that the study investigators reported that the agreed recommendations were formulated as an action plan and that there were designated persons responsible for implementation of this plan; and (8) 'follow-up' has taken place to assess whether the actions have been implemented, and to assess the patient's experience with these actions.

\subsection{Data Synthesis and Analysis}

For each trial, we extracted data on the primary outcome, 'implementation rate'. When the implementation rate was not present, we derived this rate from the percentage of DRPs resolved or the decrease in the number of potentially inappropriate prescriptions (PIPs). Trial quality and key elements of interventions were assessed independently by the two investigators (H.K. and L.B.) for each included study. Differences were resolved by discussion, or a third investigator (either A.F. or M.B.) was consulted.

Meta-regression analysis was used to assess the association between the number of key elements and the implementation rate, with the number of recommendations in the different studies as possible effect moderator. This mixedeffects analysis was conducted using the 'metafor' statistical package in R (version 2.12.2, R Project for Statistical Computing, Vienna, Austria, 2011, http://www.R-project.org).

\section{Results}

\subsection{Search Results}

A total of 620 titles were identified, 16 of which (describing 14 RCTs) met the inclusion criteria and were included in this review (Fig. 1) [3, 15-17, 19, 21-32].

\subsection{Quality Assessment of Studies}

The methodological quality of 12 of the 14 studies was assessed as adequate (i.e. 6 or more of 11 items scored 'yes') 3, 15-17, 19, 21, 23-27, 29-32] (see Table S1 in Appendix S2 [Online Resource 1]). The quality of the trial 


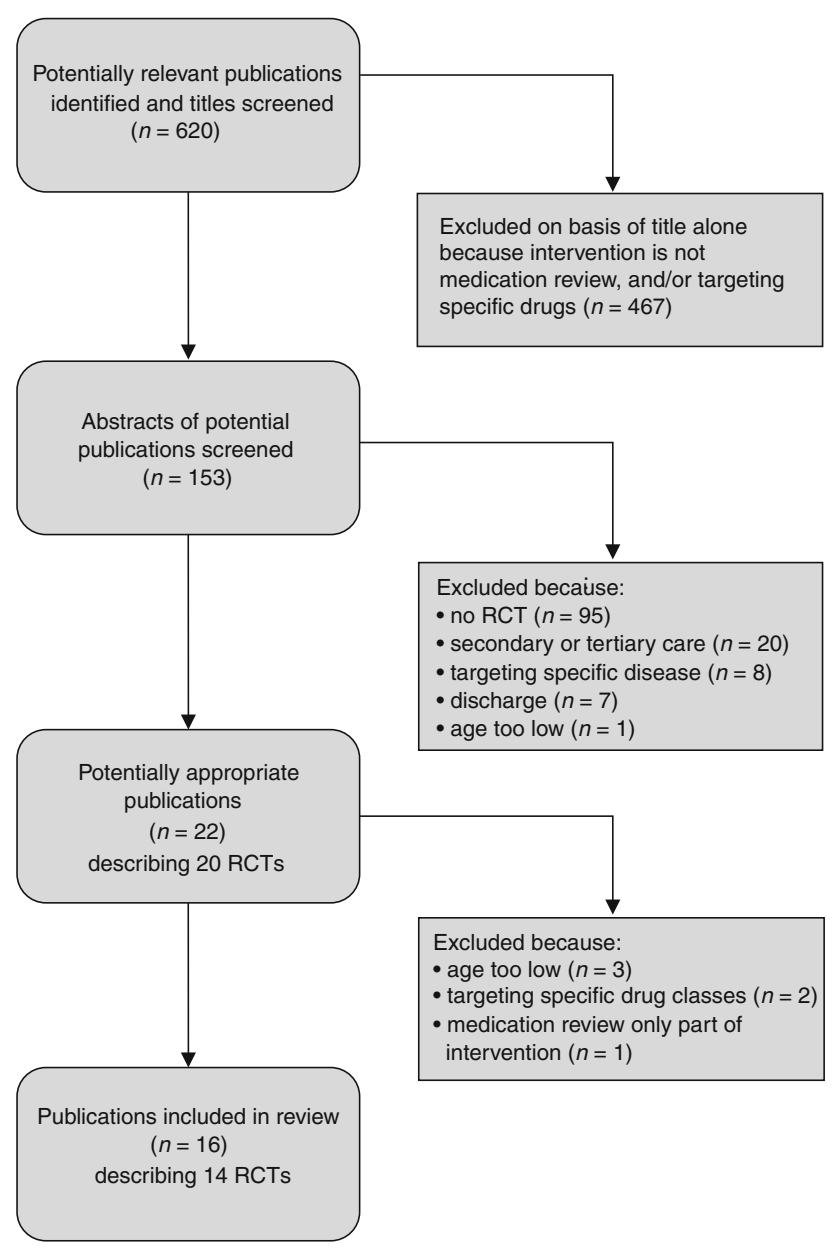

Fig. 1 Flow chart describing study selection and excluded studies. RCT randomized, controlled trial

in the two remaining studies was scored as 'low' [22, 28] and they were therefore excluded from further analysis. These studies were related; one trial formed part of a larger co-ordinated project with more countries, which was described in the other paper $[22,28]$.

In all included studies, a method of randomization was performed and eligibility criteria were specified. The majority of studies reported a method of treatment allocation [3, 15-17, 22-26, 29-31], while in three studies this was either not clearly described or not conducted [19, 21, 27, 32]. All except two studies reported similar groups at baseline [28-30]. An independent outcome assessor who was blinded to the intervention allocation was clearly described in only two studies $[17,21]$. Because of the nature of the studied intervention, the care provider was never blinded to the intervention allocation. The patient was blinded for the intervention allocation in three studies $[3,15,16,23]$. In two of these studies, patient interviews were conducted for both the intervention and the control group, but a pharmaceutical care plan was implemented only for the intervention group $[3,23]$. In the third study, no patient interview was conducted and there was no description of patient involvement with the study $[15,16]$. Point estimates and measures of variability were described in all studies. Intention-to-treat analysis was conducted in five studies $[19,21,23,25,27,32]$. The withdrawal rate was likely to have caused bias in 3 of 14 studies $[3,15,16$, 19, 21, 23-27, 29-32].

\subsection{Study Characteristics}

In Table 1, the study characteristics and outcomes are presented for the 12 included studies. The number of participants in these studies ranged from 118 to 1,188 . The mean age of the participants was 76.6 years (range 71.8-84.3) and $66 \%$ were females (range 56-90). The mean number of prescribed drugs was 7.2 (range 4.5-12). Three of these studies were performed in the US [23, 29$31]$, three in the UK $[3,19,25,32]$ and two in The Netherlands [15, 16, 24].

\subsubsection{Outcomes}

Seven of 12 studies provided data on clinical outcomes [3, 17, 19, 25-27, 29, 32]. Six of these studies reported on quality of life measured using the 36-item Short Form Health Survey (SF-36) [3, 17, 19, 26, 27, 29, 32] or the EuroQoL-5D/visual analogue scale (VAS) [25]. No effects were found on total scores for quality of life, and one study reported only negative effects on some domains in one study [17]. Data on hospital admissions were provided by four studies [19, 25-27, 32], and no significant effects were reported. Twelve studies provided data on intermediate outcomes. Two studies reported on adherence, either self-reported $[29,30]$ or measured by refill rate [23], with no effect. Two studies reported on DRPs in both the intervention and the control group, with positive effects on DRPs resolved [3, 24]. Two studies $[17,21]$ reported on potentially inappropriate medications (PIMs), with positive effects for one study [17]. Process outcomes were reported in all studies. Two studies reported a reduction in the number of (prescribed) drugs [19, 31, 32], while in four studies no effect was reported [21, 23, 25, 26]. Five studies reported an increase in the number of drug changes [15$17,19,24,31,32]$.

Implementation rates of recommendations in the intervention group are shown in Table 2. The percentage of implemented recommendations was reported in seven studies [15-17, 19, 23, 25-27, 32], while a percentage of resolved DRPs was mentioned in three studies [3, 24, 29, 30]. In two studies, the implementation rate was derived from the decrease in the number of PIPs compared with the total number of PIPs [21, 31]. 


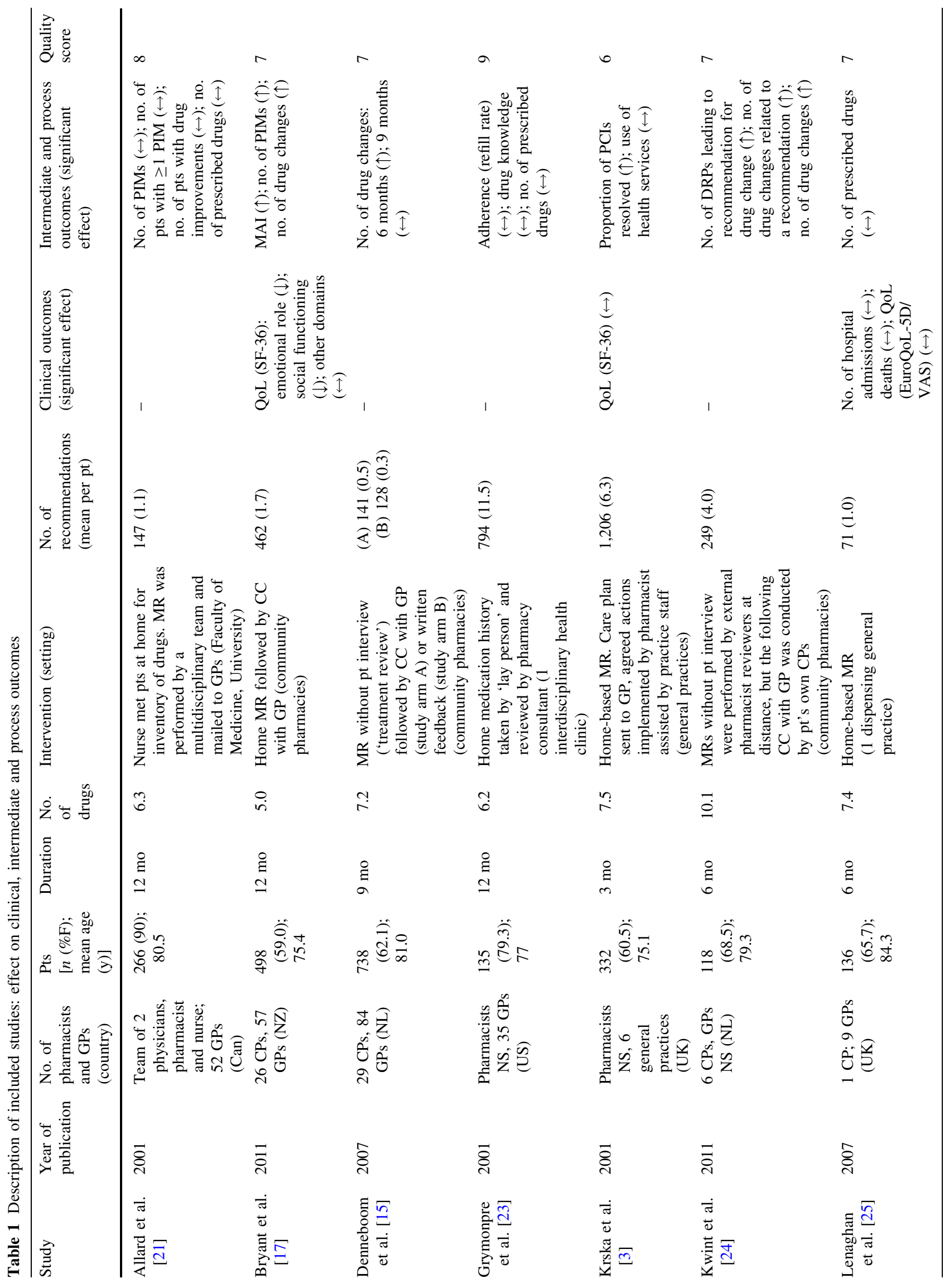




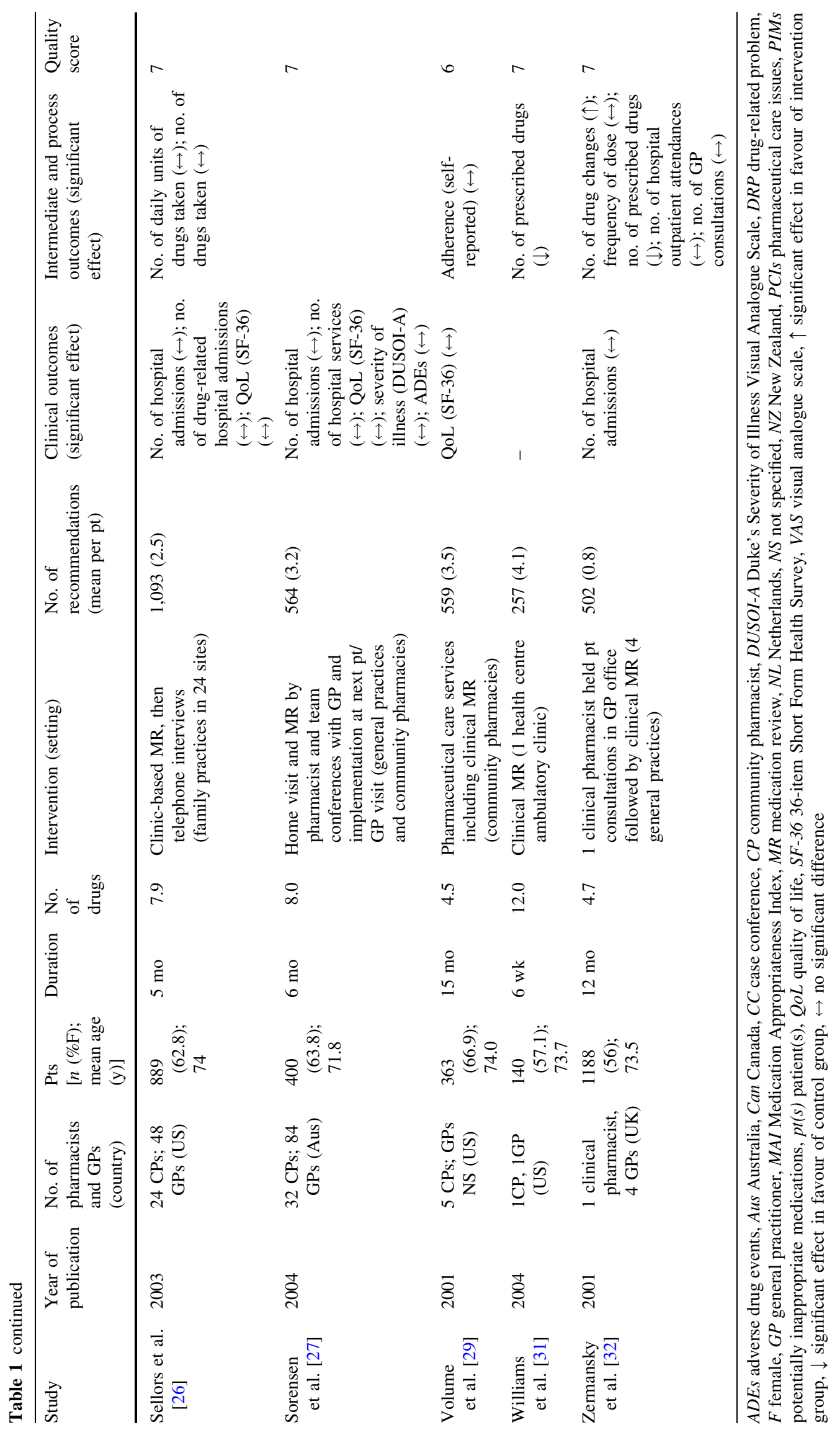




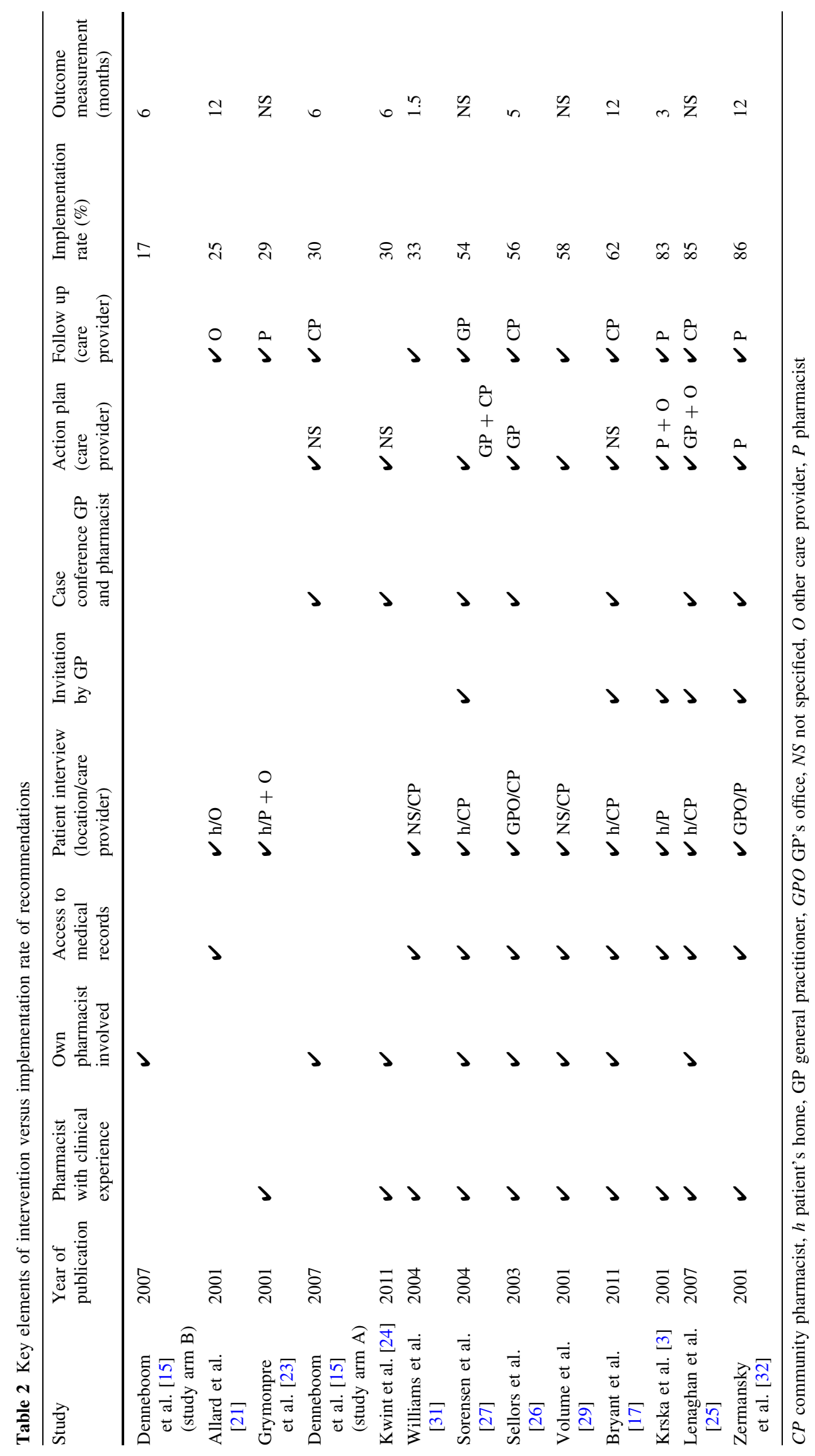




\subsubsection{Key Elements of the Intervention}

Key elements of 13 interventions from the 12 studies are shown in Table 2 . One study compared outcomes between two intervention groups (case conference and written feedback $[15,16]$ ), and therefore both study arms (A and B) are shown.

Pharmacists had clinical experience in 10 of the 13 interventions [3, 17, 19, 23, 25-27, 29-31]. Pharmacists were accredited pharmacists $[17,27]$, consultant pharmacists $[23,31]$ or clinical pharmacists $[19,32]$. They followed a university accredited externship programme [26], were clinically trained [3], experienced in medication reviews $[24,25]$ or had a post-graduate qualification in pharmacy practice [25]. In 8 of 13 interventions, the patient's own GP was involved [15-17, 25-27, 29, 30]. In the other interventions, the study pharmacist had no existing therapeutic relationship with the patient or this was not described [3, 19, 21, 23, 31, 32] (see also Table 1). Pharmacists had full access to GP's medical records of the patient in 8 of the 13 interventions $[3,17,19,25-27,29$, 32]. Patient interviews were conducted in 11 of 13 interventions, at home $[3,17,21,23,25,27]$ or in the GP's office or clinic [19, 26, 31, 32]. In 3 of 13 interventions, eligible patients were invited by the GP to participate in the study [17, 19, 27, 32]. Case conferences between GPs and pharmacists were conducted in 7 of 13 interventions [1517, 19, 24-27, 32]. In three interventions, letters with recommendations or care plans were sent to the GPs ('written feedback') [3, 15, 16, 23]. As mentioned earlier, one study compared the process outcomes of case conferences with written feedback $[15,16]$. As part of two interventions, case conferences were held by external multidisciplinary teams without the patient's own GP [21, 31] and recommendations were mailed to the GP [21] or implemented with endorsement of the GP [31]. Action plans were used for implementation of agreed recommendations in 9 of 13 interventions [3, 15-17, 19, 24-27, 29, 32]. A follow-up of the implementation of actions was described in 11 of 13 interventions [3, 15-17, $19,21,23,25-27,29,31,32]$, most often conducted by a pharmacist $[15,17,19,23,25,26,31,32]$.

\subsection{Association between Number of Key Elements and Outcomes}

Key elements of the intervention and the implementation rate are shown in Table 2 . The mean number of key elements present in the interventions was 5.2 (range 1-8). The mean implementation rate was $50 \%$ (range 17-86).

The association between the number of key elements present in the intervention and the implementation rate of recommendations was positive: an increase in number of key elements was related to an increase in implementation rate, $\beta=0.085$ (95\% CI 0.052-0.128; $p<0.0001$ ) (Fig. 2). Figure 3 is a forest plot showing the observed and expected implementation rates, estimated on the metaanalysis association between number of key elements and the implementation rate. In all but three interventions, the observed implementation rate was within the $95 \%$ confidence interval of the expected value [3, 19, 27, 32].

No meta-regression analyses were possible for the association between the number of key elements and the number of hospital admissions ( $n=4$ studies), the number of drug changes ( $n=5$ studies) and the number of prescribed drugs ( $n=5$ studies), because of the low number of studies and participants with these outcomes.

\section{Discussion}

This systematic review shows a significant association between the number of key elements of the intervention reflecting collaborative aspects in medication review and
Fig. 2 Bubble plot of number of key elements of intervention vs. implementation rate of recommendations. The size of the circles reflects the number of recommendations in the intervention group of the different studies $[3,15,17,21$, 23-27, 29, 31, 32]

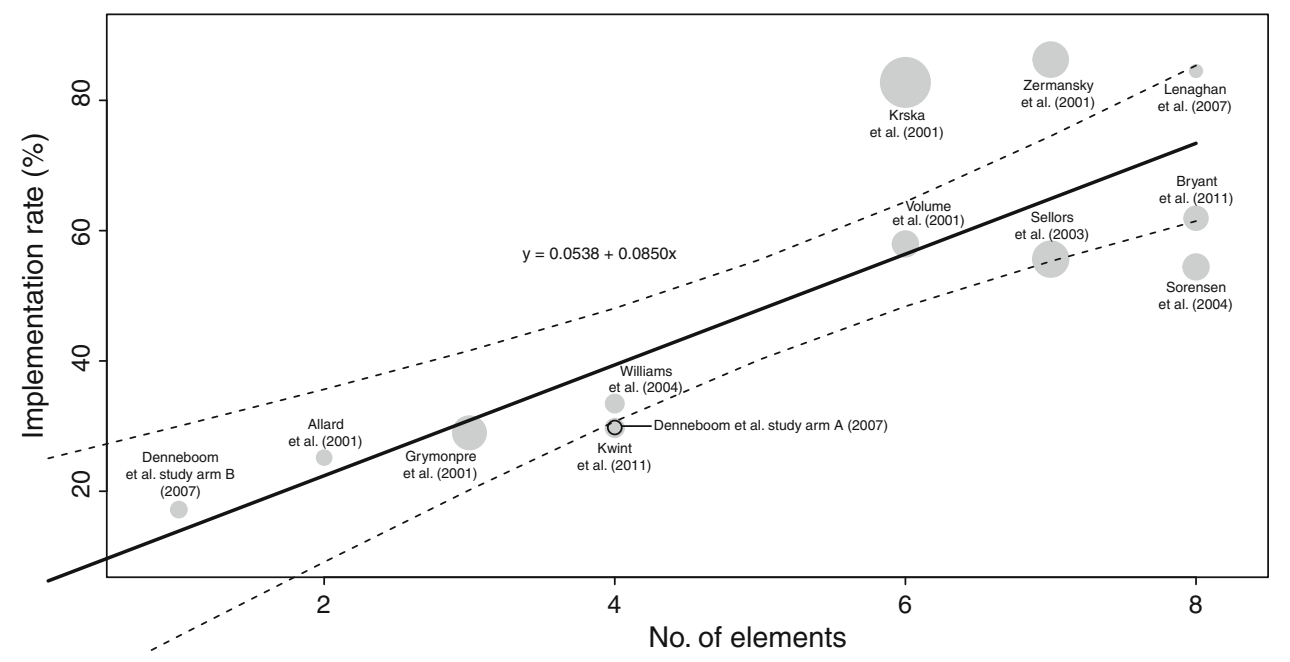


Fig. 3 Forest plot of observed and expected implementation rate of recommendations $[3,15$, 17, 21, 23-27, 29, 31, 32]. Mean observed implementation rate with confidence intervals (95\% CIs). The grey diamonds represent the CIs of the expected implementation rate estimated on the metaregression analysis association elements and the implementation rate between the number of key

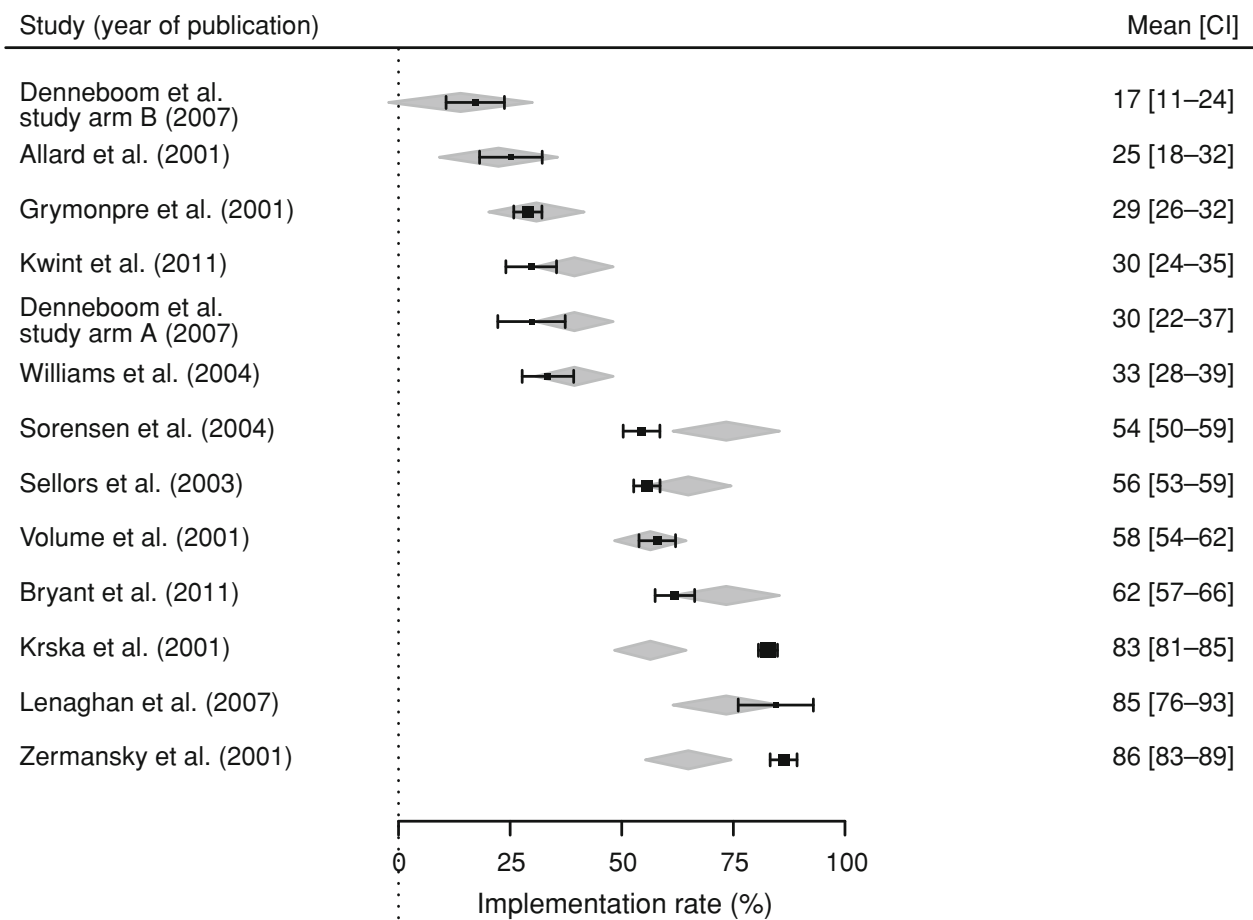

the implementation rate. This suggests that more intensive collaboration between GP and pharmacist in medication review leads to higher recommendation implementation rates.

The expected implementation rate could be predicted from the number of key elements estimated from this association (Fig. 3). This model gives a good prediction of the implementation rate for the majority of the studies. For three studies, the expected implementation rate was different from the observed value [3, 19, 27, 32]. A higher implementation rate than expected was observed in the studies by Krska et al. [3] and Zermansky et al. [19, 32]. In the study by Zermansky et al. [19, 32], one pharmacist collaborated with only a few GPs, similar to the study by Lenaghan et al. [25], resulting in comparably high implementation rates. The major difference with the Lenaghan study was that the patient's own pharmacist was not involved. However, it is conceivable that this (clinical) pharmacist established a good relationship with this small number of GPs and their patients whilst he was consulting patients in the GP's office. Studies with pharmacists working at a GP practice yielded high rates of acceptance of recommendations [18, 19, 26, 32]. Conversely, Krska et al. [3] was the only study with a high implementation rate without a case conference. This face-toface meeting between GPs and pharmacists to discuss the pharmacist's recommendations is often considered one of the most important and key elements of the collaborative approach in medication review $[13,15,16,33]$. In the study by Krska et al. [3], pharmacists were assisted by practice staff in the implementation of accepted actions. Possibly, this partly explains the high implementation rate. Furthermore, the nature and number of pharmacists and their relationship with patients was not specified [3]. On the other hand, a lower than expected implementation rate was observed in the intervention by Sorensen et al. [27]. In this study, a large number of pharmacists collaborated with an even larger number of GPs, which could have made it difficult to achieve high implementation rates. The implementation rate and numbers of GPs and pharmacists in the study of Sorensen et al. [27] were similar to those in the study by Sellors et al. [26]. The major difference was that, in the study of Sorensen et al. [27], patients were invited by the GP.

There have been no earlier systematic reviews investifound a significant association between the number of key elements reflecting collaborative aspects and the implementation rate. This finding is in agreement with other medication review studies in secondary and tertiary care where direct communication between healthcare providers revealed higher acceptance rates of recommendations [34, 35]. For clinical and intermediate outcomes, no association could be assessed, because the number of studies reporting these outcomes was too low. Earlier systematic reviews reported no effect on hospital admissions and quality of life $[8,10]$. Compared with these reviews, our scope was more focused, as we included only RCTs, home-dwelling patients in primary care, a mean age of 70 years and no recent discharge, yielding only 12 trials after quality gating the implementation rate of recommendations. We 
assessment. However, due to our inclusion criteria, patients in our review were relatively healthy and not directly at risk for hospital admission. In contrast, studies on medication review in hospitals were more successful in preventing hospital (re)admissions because they generally reviewed patients who were admitted to hospital and at high risk for readmission [36, 37].

There may be additional reasons for low implementation rates. Sellors et al. [26] showed that these reasons might include patient reluctance, previous failed attempts at the same strategy and a relatively short period for implementation combined with the occurrence of more urgent issues. In particular, the periods over which implementation rates were measured varied between the different studies in this review. Nor do we know if the recommendations in the different studies were clinically appropriate [17]. GP's perceptions of pharmacists' recommendations in the GPPC study revealed that they generally found the recommendations useful although at times theoretical [38]. Pharmacist's recommendations may be less appropriate if a high proportion of patients are already receiving the recommended treatment, for example, in the MEDMAN study ('ceiling effect') [39].

There were several strengths to this study. First of all, like in other systematic reviews on medication review, trials reported very heterogeneous outcomes that could not be pooled. In this systematic review, we could compare different trials using the implementation rate as the common (process) outcome. Implementation rates are also reported in many (observational) studies in home-dwelling patients $[40,41]$ as well as for patients in nursing homes $[34,42]$. Implementation rate has a greater significance than acceptance rate of pharmacist's recommendations by physicians because it includes enactment of the recommendation by a care provider and the level of acceptance of recommendations by the patient. Secondly, we described eight different elements of the intervention that reflect collaborative aspects between GPs and pharmacists. These key elements were based on described facilitators and barriers in medication review [12-14]. Thirdly, the importance of patient involvement in medication review was also reflected by the key elements 'own pharmacist involved', 'patient interview' and 'follow-up'.

Our decision to consider all eight key elements of the intervention as equivalent weighted determinants for the implementation rate could be seen as a limitation. For example, the face-to-face discussion between pharmacists and GPs seemed a key element that could have more weight. The small number of studies precluded us from studying the association of the individual key elements in a multivariate design. Also, there could have been other key elements reflecting collaborative aspects that we may have missed. Furthermore, it was not possible to discriminate between the clinical relevance of the implemented recommendations in the different studies. This clinical relevance was only described in the study by Denneboom et al. $[15,16]$. Finally, it cannot be ruled out that some relevant RCTs may have been missed or excluded.

\section{Conclusion}

This systematic review showed that the number of key elements reflecting collaborative aspects of medication review was significantly associated with the recommendation implementation rate. Further clinical trials could demonstrate whether an increase in collaborative aspects leads to higher implementation rates.

Based on this model, future studies for elderly in primary care could consider these key elements of intervention to design a standardized medication review process. More research is needed to assess which key elements of this collaborative approach are the most important and if there are additional elements that may influence implementation rates. Next to the physician and the pharmacist, the patient is the third main player in the medication review process. Future studies could focus on the influence of the patient on the implementation rate. Large multicentre trials in primary care are needed to draw definitive conclusions on whether a standardized collaborative approach in medication review could affect clinical outcomes. Such trials may be expensive, difficult to organize in practice settings and it may be questioned how many and which elderly home-dwelling patients in primary care are at greatest risk for negative clinical outcomes.

Acknowledgments No funds were received for conducting this review. All authors declare that they have no conflict of interests that are directly relevant to the content of this study. The authors thank J.C. Riemens-Louisse, student, for her contribution to the data extraction forms and quality assessment forms and S.V. Belitser for her contribution to the statistical analysis.

\section{References}

1. Rollason V, Vogt N. Reduction of polypharmacy in the elderly: a systematic review of the role of the pharmacist. Drugs Aging. 2003;20:817-32.

2. Castelino RL, Bajorek BV, Chen TF. Targeting suboptimal prescribing in the elderly: a review of the impact of pharmacy services. Ann Pharmacother. 2009;43:1096-106.

3. Krska J, Cromarty JA, Arris F, Jamieson D, Hansford D, Duffus PRS, et al. Pharmacist-led medication review in patients over 65: a randomized, controlled trial in primary care. Age Ageing. 2001;30:205-11.

4. Hanlon JT, Schmader KE, Ruby CM, Weinberger M. Suboptimal prescribing in older inpatients and outpatients. J Am Geriatr Soc. 2001;49:200-9. 
5. Shaw J, Seal R, Pilling M. Task force on medicines partnership and the national collaborative medicines management services programme. Room for review: a guide to medication review. 2002. http://www.npc.nhs.uk/review_medicines/intro/resources/ room_for_review.pdf. Accessed 21 Feb 2012.

6. Clyne W, Blenkinsopp A, Seal R. National prescribing centre. A guide to medication review. 2008. http://www.npc.nhs.uk/review _medicines/intro/resources/agtmr_web1.pdf. Accessed 22 Feb 2012.

7. Lowe CJ, Petty DR, Zermansky AG, Raynor DK. Development of a method for clinical medication review by a pharmacist in general practice. Pharm World Sci. 2000;22:121-6.

8. Holland R, Desborough J, Goodyer L, Hall S, Wright D, Loke YK. Does pharmacist-led medication review help to reduce hospital admissions and deaths in older people? A systematic review and meta-analysis. Br J Clin Pharmacol. 2008;65:303-16.

9. Royal S, Smeaton L, Avery AJ, Hurwitz B, Sheikh A. Interventions in primary care to reduce medication related adverse events and hospital admissions: systematic review and meta-analysis. Qual Saf Health Care. 2006;15:23-31.

10. Nkansah N, Mostovetsky O, Yu C, Chheng T, Beney J, Bond $\mathrm{CM}$, et al. Effect of outpatient pharmacists' non-dispensing roles on patient outcomes and prescribing patterns. Cochrane Database Syst Rev 2010;(7):CD000336.

11. Holland R, Smith R, Harvey I. Where now for pharmacist led medication review? J Epidemiol Community Health. 2006;60:92-3.

12. Urbis Keys Young. Evaluation of the home medicines review program (pharmacy component): final report. 2005. http://www. beta.guild.org.au/uploadedfiles/Medication_Management_Reviews/ Overview/Urbis\%20Keys\%20Young\%20evaluation.pdf. Accessed $21 \mathrm{Feb} 2012$

13. MacKeigan LD, Nissen LM. Clinical pharmacy services in the home. Dis Manage Health Outcomes. 2008;16:227-44.

14. Niquille A, Lattman C, Bugnon O. Medication reviews led by community pharmacists in Switzerland: a qualitative survey to evaluate barriers and facilitators. Pharm Pract. 2010;8:35-42.

15. Denneboom W, Dautzenberg MG, Grol R, De Smet PA. Treatment reviews of older people on polypharmacy in primary care: cluster controlled trial comparing two approaches. Br J Gen Pract. 2007;57:723-31.

16. Denneboom W, Dautzenberg MGH, Grol R, De Smet PAGM. Comparison of two methods for performing treatment reviews by pharmacists and general practitioners for home-dwelling elderly people. J Eval Clin Pract. 2008;14:446-52.

17. Bryant LJ, Coster G, Gamble GD, McCormick RN. The General Practitioner-Pharmacist Collaboration (GPPC) study: a randomised controlled trial of clinical medication reviews in community pharmacy. Int J Pharm Pract. 2011;19:94-105.

18. Isetts BJ, Brown LM, Schondelmeyer SW, Lenarz LA. Quality assessment of a collaborative approach for decreasing drugrelated morbidity and achieving therapeutic goals. Arch Intern Med. 2003;163:1813-20.

19. Zermansky AG, Petty DR, Raynor DK, Lowe CJ, Freemantle N, Vail A. Clinical medication review by a pharmacist of patients on repeat prescriptions in general practice: a randomised controlled trial. Health Technol Assess. 2002;6:1-86.

20. Verhagen AP, de Vet HC, de Bie RA, Kessels AG, Boers M, Bouter LM, et al. The Delphi list: a criteria list for quality assessment of randomized clinical trials for conducting systematic reviews developed by Delphi consensus. J Clin Epidemiol. 1998;51:1235-41.

21. Allard J, Hebert R, Rioux M, Asselin J, Voyer L. Efficacy of a clinical medication review on the number of potentially inappropriate prescriptions prescribed for community-dwelling elderly people. CMAJ. 2001;164:1291-6.
22. Bernsten C, Bjorkman I, Caramona M, Crealey G, Frokjaer B, Grundberger E, et al. Improving the well-being of elderly patients via community pharmacy-based provision of pharmaceutical care: a multicentre study in seven European countries. Drugs Aging. 2001;18:63-77.

23. Grymonpre RE, Williamson DA, Montgomery PR. Impact of a pharmaceutical care model for non-institutionalised elderly: results of a randomised, controlled trial. Int $\mathbf{J}$ Pharm Practice. 2001;9:235-41.

24. Kwint HF, Faber A, Gussekloo J, Bouvy ML. Effects of medication review on drug-related problems in patients using automated drug-dispensing systems: a pragmatic randomized controlled study. Drugs Aging. 2011;28:305-14.

25. Lenaghan E, Holland R, Brooks A. Home-based medication review in a high risk elderly population in primary care-The POLYMED randomised controlled trial. Age Ageing. 2007;36:292-7.

26. Sellors J, Kaczorowski J, Sellors C, Dolovich L, Woodward C, Willan A, et al. A randomized controlled trial of a pharmacist consultation program for family physicians and their elderly patients. CMAJ. 2003;169:17-22.

27. Sorensen L, Stokes JA, Purdie DM, Woodward M, Elliott R, Roberts MS. Medication reviews in the community: results of a randomized, controlled effectiveness trial. Br J Clin Pharmacol. 2004;58:648-64.

28. Sturgess IK, McElnay JC, Hughes CM, Crealey G. Community pharmacy based provision of pharmaceutical care to older patients. Pharm World Sci. 2003;25:218-26.

29. Volume CI, Farris KB, Kassam R, Cox CE, Cave A. Pharmaceutical care research and education project: patient outcomes. J Am Pharm Assoc. 2001;41:411-20.

30. Kassam R, Farris KB, Burback L, Volume CI, Cox CE, Cave A. Pharmaceutical care research and education project: pharmacists' interventions. J Am Pharm Assoc. 2001;41:401-10.

31. Williams ME, Pulliam CC, Hunter R, Johnson TM, Owens JE, Kincaid J, et al. The short-term effect of interdisciplinary medication review on function and cost in ambulatory elderly people. J Am Geriatr Soc. 2004;52:93-8.

32. Zermansky AG, Petty DR, Raynor DK, Freemantle N, Vail A, Lowe CJ. Randomised controlled trial of clinical medication review by a pharmacist of elderly patients receiving repeat prescriptions in general practice. BMJ. 2001;323:1340-3.

33. Almeida Neto AC, Chen TF. Exploring elements of interprofessional collaboration between pharmacists and physicians in medication review. Pharm World Sci. 2007;29:574-6.

34. Nishtala PS, McLachlan AJ, Bell JS, Chen TF. A retrospective study of drug-related problems in Australian aged care homes: medication reviews involving pharmacists and general practitioners. J Eval Clin Pract. 2011;17:97-103.

35. Spinewine A, Swine C, Dhillon S, Lambert P, Nachega JB, Wilmotte L, et al. Effect of a collaborative approach on the quality of prescribing for geriatric inpatients: a randomized, controlled trial. J Am Geriatr Soc. 2007;55:658-65.

36. Gillespie U, Alassaad A, Henrohn D, Garmo H, HammarlundUdenaes $\mathrm{M}$, Toss $\mathrm{H}$, et al. A comprehensive pharmacist intervention to reduce morbidity in patients 80 years or older: a randomized controlled trial. Arch Intern Med. 2009;169: 894-900.

37. Makowsky MJ, Koshman SL, Midodzi WK, Tsuyuki RT. Capturing outcomes of clinical activities performed by a rounding pharmacist practicing in a team environment: the COLLABORATE study. Med Care. 2009;47:642-50.

38. Bryant L, Coster G, McCormick R. General practitioner perceptions of clinical medication reviews undertaken by community pharmacists. J Prim Health Care. 2010;2:225-33. 
39. Bond C. The MEDMAN study: a randomized controlled trial of community pharmacy-led medicines management for patients with coronary heart disease. Fam Pract. 2007;24:189-200.

40. Farris KB, Cote I, Feeny D, Johnson JA, Tsuyuki RT, Brilliant S, et al. Enhancing primary care for complex patients: demonstration project using multidisciplinary teams. Can Fam Physician. 2004;50:998-1003.
41. Krass I, Smith C. Impact of medication regimen reviews performed by community pharmacists for ambulatory patients through liaisons with general medical practitioners. Int J Pharm Pract. 2000;8:111-20.

42. Halvorsen KH, Ruths S, Granas AG, Viktil KK. Multidisciplinary intervention to identify and resolve drug-related problems in Norwegian nursing homes. Scand J Prim Health Care. 2010;28:82-8. 